\title{
INFINITE NON-LINEAR PROGRAMMING
}

\author{
M. A. HANSON \\ (received 10 September 1962)
}

\section{Introduction}

In recent years there has been extensive development in the theory and techniques of mathematical programming in finite spaces. It would be very useful in practice to extend this development to infinite spaces, in order to treat more realistically the problems that arise for example in economic situations involving infinitely divisible processes, and in particular problems involving time as a continuous variable. A more mathematical reason for seeking such generalisation is possibly that of obtaining a unification of mathematical programming with other branches of mathematics concerned with extrema, such as the calculus of variations.

Some early results in infinite programming were obtained by Duffin [1] who investigated infinite linear programmes. In the present paper the duality theorems of convex programming are formulated in Banach spaces, based on Hurwicz' generalisation [2] of the Kuhn-Tucker theorem. Duality theorems of varying generality in finite Euclidean spaces have been developed by Kuhn and Tucker [3], Karlin [4], Dennis [5], Dorn [6], [7], Wolfe [8], and Hanson [9].

\section{Notation and definitions}

Let $x$ denote an element of the set $X$ in a Banach space $\mathscr{X}$. The conjugate, or dual, space of $\mathscr{X}$ will be denoted by $\mathscr{X}^{*}$ whose typical element is $x^{*}$. Positive elements of $\mathscr{X}$ are defined to be the elements of some specified convex cone $P_{x} \subset \mathscr{X}$. It will be assumed that $P_{x}$ contains the origin, which will be denoted by 0 for all spaces.

Three ordering relationships are subsequently defined:

and

$$
\begin{aligned}
& x_{1} \geqq x_{2} \quad \text { means that } x_{1}-x_{2} \in P_{x} \\
& x_{1} \geq x_{2} \quad \text { means that } x_{1} \geqq x_{2} \text { but } x_{2} \geqq x_{1}
\end{aligned}
$$

$$
x_{1}>x_{2} \text { means that } x_{1}-x_{2} \in \text { Int. } P_{x} .
$$

In the conjugate space the expression $x_{0}^{*} \geqq 0$ means 


$$
x_{0}^{*}(x) \geqq 0 \text { for all } x \geqq 0 .
$$

A programme in Banach space is represented by the couple $\langle t, g\rangle$ where $f$ and $g$ are defined on $X \subset \mathscr{X}$ into the Banach spaces $\mathscr{Y}$ and $\mathscr{Z}$ respectively; and a maximal value of the programme is defined to be an element $y_{0}$ of the set $Y=f\left(P_{x} \cap g^{-1}\left(P_{z}\right)\right)$ such that for $y \in Y, y \geqq y_{0}$ implies $y \leqq y_{0}$. Such an element $y_{0}$ is said to be maximal over $Y$, and if $y_{0}=f\left(x_{0}\right)$ then $x_{0}$ is said to maximise $f(x)$ over $f\left(P_{x} \cap g^{-1}\left(P_{z}\right)\right)$. (It should be mentioned that the simultaneous inequalities $x \geqq y$ and $y \geqq x$ do not necessarily imply that $x=y$.) In the terminology of economics the spaces $\mathscr{X}, \mathscr{Y}, \mathscr{Z}$ are called the activity, objective, and constraint spaces respectively. The value $x_{0}$ is called the optimal activity, and $y_{0}$ the optimal value of the programme.

The symbol $\delta f\left(x_{0} ; x^{1}\right)$ will denote the Fréchet differential of $f(x)$ at $x_{0}$ with increment $x^{1}$.

A concave function $f: X \rightarrow \mathscr{Y}$ is such that

$$
f\left(\lambda x_{1}+(1-\lambda) x_{2}\right) \geqq \lambda f\left(x_{1}\right)+(1-\lambda) f\left(x_{2}\right) \text { for all } x_{1}, x_{2} \text { in } X
$$

and $0 \leqq \lambda \leqq 1$. It follows that if $f$ is differentiable then

$$
f\left(x_{1}\right) \leqq f\left(x_{2}\right)+\delta f\left(x_{2} ; x_{1}-x_{2}\right) .
$$

A convex function has the relevant above inequalities reversed.

\section{The dual problem}

Let $f$ and $g$ be Fréchet differentiable functions on the Banach space $\mathscr{X}$ into the Banach spaces $\mathscr{Y}$ and $\mathscr{Z}$ respectively, and let $x_{0}$ maximise the programme $\langle t, g\rangle$. In the problems to be considered it will be assumed that the constraints of the programme satisfy Hurwicz' generalisation of the Kuhn-Tucker constraint conditions, namely that $g$ is regular ([2], p. 95) and there is regular convexity $([2]$, p. 61) of the set

where

$$
\left\{w^{*}: w^{*}=T^{*}\left(v^{*}\right), v^{*} \geqq 0, v \in \mathscr{Z} \times \mathscr{W}\right\}
$$

and

$$
\mathscr{W}=\{w: w=(\rho, x), \rho \text { real, } x \in \mathscr{X}\}
$$

$$
T(\rho, x)=\left[\delta g\left(x_{0} ; x\right)-\rho\left(\delta g\left(x_{0} ; x_{0}\right)-g\left(x_{0}\right)\right),(\rho, x)\right] .
$$

Further the space $\mathscr{Y}$ will be restricted to be such that to each closed convex cone in $\mathscr{Y}$ there exists a continuous linear functional $y^{*} \in \mathscr{Y}^{*}$ which is strictly positive on this cone.

Define the Lagrangian expression

$$
\Phi\left(x, z^{*}\right)=y_{0}^{*}(f(x))+z^{*}(g(x)) .
$$

Hurwicz has shown ([2] Theorem V.3.3.4.) that for $\%$ in $y_{0}^{*}>0$ there exists 
$z_{0}^{*} \geqq 0$ such that $\Phi\left(x, z^{*}\right)$ has a non-negative quasi-saddle-point at $\left(x_{0}, z_{0}^{*}\right.$; $\left.y_{0}^{*}\right)$; that is, the following relations hold:

$$
\begin{aligned}
& \delta_{x} \Phi\left(\left(x_{0}, z_{0}^{*}\right) ; x\right) \leqq 0 \text { for all } x \geqq 0 \\
& \delta_{x} \Phi\left(\left(x_{0}, z_{0}^{*}\right) ; x_{0}\right)=0 \\
& \delta_{z}^{*} \Phi\left(\left(x_{0}, z_{0}^{*}\right) ; z^{*}\right) \geqq 0 \text { for all } z^{*} \geqq 0 \\
& \delta_{z}^{*} \Phi\left(\left(x_{0}, z_{0}^{*}\right) ; z_{0}^{*}\right)=0 .
\end{aligned}
$$

These results will be used to establish a dual programme to the programme $\langle f, g\rangle$ in the case where $f$ and $g$ are differentiable concave functions.

The activity, objective, and constraint spaces of the dual programme will be denoted by $\mathscr{S}, \mathscr{T}$, and $\mathscr{U}$ respectively, defined by their elements:

$$
\begin{aligned}
s \in \mathscr{S} & =\mathscr{X} \times \mathscr{Z}^{*} \\
t(s) & =\Phi\left(x, z^{*}\right)-\delta_{x} \Phi\left(\left(x, z^{*}\right) ; x\right) \\
u(s) & =-\delta_{x} \Phi\left(\left(x, z^{*}\right) ; x^{1}\right)
\end{aligned}
$$

where $u(s)$ is defined for elements $x^{1} \in P_{x}$.

It will be shown that the dual problem is to find $s_{0} \in \mathscr{P}$ which minimises $t(s)$ over $t\left(P_{s} \cap u^{-1}\left(P_{u}\right)\right)$ where $P_{s}=P_{x} \times P_{z}^{*}$; and that a solution of either primal or dual problem, if such exists, implies the existence, under conditions to be stated, of a solution of the other problem, the optimal values of primal and dual objectives are the same, and the optimal activity $x_{0}$ of the primal is a component of the optimal activity $s_{0}=\left(x_{0}, z_{0}^{*}\right)$ of the dual. The evaluation of $s_{0}$ may also of course be considered as a maximisation problem, namely, the programme $\langle-t, u\rangle$.

THEOREM 1. If $f$ and $g$ are Fréchet differentiable concave functions and if for some $y_{0}^{*}>0$ there exists $x_{0}$ which maximises $y_{0}^{*}(f(x))$ over $y_{0}^{*}\left(f\left(P_{x} \cap g^{-1}\left(P_{z}\right)\right)\right)$ then there exists $z_{0}^{*}$ such that $s_{0}=\left(x_{0}, z_{0}^{*}\right)$ minimises $t(s)$ over $t\left(P_{s} \cap u^{-1}\left(P_{u}\right)\right)$, and $y_{0}^{*}\left(f\left(x_{0}\right)\right)=t\left(s_{0}\right)$.

PROOF. Let $z_{0}^{*}$ be the functional introduced in the expressions $(1)$ to (4). Then $s_{0}=\left(x_{0}, z_{0}^{*}\right)$ satisfies the dual constraints. Let $s=\left(x, z^{*}\right)$ be any other element of $\mathscr{S}$ satisfying the constraints. Then

$$
\begin{aligned}
t\left(s_{0}\right)-t(s) & =\Phi\left(x_{0}, z_{0}^{*}\right)-\delta_{x} \Phi\left(\left(x_{0}, z_{0}^{*}\right) ; x_{0}\right)-\Phi\left(x, z^{*}\right)+\delta_{x} \Phi\left(\left(x, z^{*}\right) ; x\right) \\
& =\Phi\left(x_{0}, z_{0}^{*}\right)-\Phi\left(x, z^{*}\right)-\delta_{x} \Phi\left(\left(x, z^{*}\right) ; x\right) \\
& \text { using }(2), \\
& =\Phi\left(x_{0}, z_{0}^{*}\right)-\Phi\left(x_{0}, z^{*}\right)+\Phi\left(x_{0}, z^{*}\right)-\Phi\left(x, z^{*}\right)+\delta_{x} \Phi\left(\left(x, z^{*}\right) ; x\right) \\
& \leqq z_{0}^{*}\left(g\left(x_{0}\right)\right)-z^{*}\left(g\left(x_{0}\right)\right)+\delta_{x} \Phi\left(\left(x, z^{*}\right) ; x_{0}-x\right)+\delta_{x} \Phi\left(\left(x, z^{*}\right) ; x\right) \\
& \text { since } \Phi \text { is concave, } \\
& =\delta_{x} \Phi\left(\left(x, z^{*}\right) ; x_{0}\right) \\
& \text { using (3) and }(4), \\
& \leqq 0
\end{aligned}
$$


since $\left(x, z^{*}\right)$ satisfies the dual constraints. Hence $s_{0}$ minimises the dual problem.

Further, the optimal value of the dual objective function is $\Phi\left(x_{0}, z_{0}^{*}\right)-\delta_{x} \Phi\left(\left(x_{0}, z_{0}^{*}\right) ; x_{0}\right)$ which by (2) and (4) is equal to the optimal value of the primal objective function $y_{0}^{*}\left(f\left(x_{0}\right)\right)$.

Note that although this theorem has been stated for the positive cone in the $\mathscr{S}$ space defined as $P_{x} \times P_{z}^{*}$, the proof applies equally well to the wider cone $\mathscr{X} \times P_{z}^{*}$.

\section{THEOREM 2. If}

(i) $\Phi$ is continuously differentiable with respect to $x \in \mathscr{X}$

(ii) there exists for any $z^{*} \in P_{z}^{*}$ and $x^{1} \in \mathscr{X}$ a continuous one-to-one inversion of $P_{u}$ into $u^{-1}\left(P_{u}\right)$,

and

(iii) there exists $s_{0}=\left(x_{0}, z_{0}^{*}\right)$ which minimises $t(s)$ over $t\left(P_{s} \cap u^{-1} P_{u}\right)$ then $x_{0}$ maximises $y_{0}^{*}(f(x))$ over $y_{0}^{*}\left(f\left(P_{x} \cap g^{-1}\left(P_{x}\right)\right)\right)$.

Proof. It follows from the hypothesis that $\left(x_{0}, z_{0}^{*}\right)$ is a feasible solution of the problem:

$$
\begin{aligned}
& \text { minimise } \Phi\left(x_{0}, z^{*}\right)-\delta_{x} \Phi\left(\left(x, z_{0}^{*}\right) ; x\right) \\
& \text { subject to }-\delta_{x} \Phi\left(\left(x, z_{0}^{*}\right) ; x\right) \geqq 0
\end{aligned}
$$

and

$$
z^{*} \geqq 0
$$

It will be shown that $\left(x_{0}, z_{0}^{*}\right)$ is the optimum solution of the problem. Put

$$
u^{0}(x)=-\delta_{x} \Phi\left(\left(x, z_{0}^{*}\right) ; x\right) .
$$

Suppose $\left(z_{1}^{*}, u_{1}\right)$ is a feasible solution of the above problem such that

$$
\Phi\left(x_{0}, z_{1}^{*}\right)+u_{1} \leq \Phi\left(x_{0}, z_{0}^{*}\right)+u_{0}
$$

where $u_{1}$ is some particular value of $-\delta_{x} \Phi\left(\left(x, z_{0}^{*}\right) ; x\right)$ and

$$
u_{0}=-\delta_{x} \Phi\left(\left(x_{0}, z_{0}^{*}\right) ; x_{0}\right) \text {, }
$$

that is,

$$
z_{1}^{*}\left(g\left(x_{0}\right)\right)-z_{0}^{*}\left(g\left(x_{0}\right)\right)+u_{1}-u_{0} \leq 0
$$

Let

$$
z_{2}^{*}=z_{0}^{*}+k\left(z_{1}^{*}-z_{0}^{*}\right)
$$

and

$$
u_{2}=u_{0}+k\left(u_{1}-u_{0}\right)
$$

for $0<k \leqq 1$. 
Thus $\left(z_{2}^{*}, u_{2}\right)$ is a feasible solution of the problem (6), (7), (8). Therefore by hypothesis there exists $\left(x_{2}, z_{2}^{*}\right) \in P_{s} \cap u^{-1}\left(P_{u}\right)$ such that

$$
u_{2}=-\delta_{x} \Phi\left(\left(x_{2}, z_{2}^{*}\right) ; x_{0}\right)
$$

Define

$$
x_{1}=x_{0}+k^{-1}\left(x_{2}-x_{0}\right)
$$

Then

$$
\begin{gathered}
\begin{aligned}
& {\left[\Phi\left(x_{2}, z_{2}^{*}\right)-\delta_{x} \Phi\left(\left(x_{2}, z_{2}^{*}\right) ; x_{2}\right)\right]-\left[\Phi\left(x_{0}, z_{0}^{*}\right)-\delta_{x} \Phi\left(\left(x_{0}, z_{0}^{*}\right) ; x_{0}\right)\right] } \\
&=\left[\Phi\left(x_{2}, z_{2}^{*}\right)-\Phi\left(x_{0}, z_{2}^{*}\right)\right]+\left[\Phi\left(x_{0}, z_{2}^{*}\right)-\Phi\left(x_{0}, z_{0}^{*}\right)\right] \\
&-\delta_{x} \Phi\left(\left(x_{2}, z_{2}^{*}\right) ; x_{2}\right)+\delta_{x} \Phi\left(\left(x_{0}, z_{0}^{*}\right) ; x_{0}\right) \\
& \leqq \delta_{x} \Phi\left(\left(x_{0}, z_{2}^{*}\right) ; x_{2}-x_{0}\right)- \delta_{x} \Phi\left(\left(x_{2}, z_{2}^{*}\right) ; x_{2}-x_{0}\right) \\
&+ z_{2}^{*}\left(g\left(x_{0}\right)\right)-z_{0}^{*}\left(g\left(x_{0}\right)\right)+u_{2}-u_{0}
\end{aligned}
\end{gathered}
$$

since $\Phi$ is concave,

$$
\begin{aligned}
=\delta_{x} \Phi\left(\left(x_{0}, z_{2}^{*}\right) ;\right. & \left.k\left(x_{1}-x_{0}\right)\right)-\delta_{x} \Phi\left(\left(x_{0}+k\left(x_{1}-x_{0}\right), z_{2}^{*}\right) ;\right. \\
& \left.k\left(x_{1}-x_{0}\right)\right)+k z_{1}^{*}\left(g\left(x_{0}\right)\right)-k z_{0}^{*}\left(g\left(x_{0}\right)\right)+k u_{1}-k u_{0}
\end{aligned}
$$

using (13), (14) and (16).

$$
\begin{aligned}
=k\left\{\delta_{x} \Phi\left(\left(x_{0}, z_{2}^{*}\right) ; x_{1}-x_{0}\right)-\delta_{x} \Phi\right. & \left(\left(x_{0}+k\left(x_{1}-x_{0}\right) ; x_{1}-x_{0}\right)\right. \\
& \left.+z_{1}^{*}\left(g\left(x_{0}\right)\right)-z_{0}^{*}\left(g\left(x_{0}\right)\right)+u_{1}-u_{0}\right\}
\end{aligned}
$$

since the Fréchet differential is linear in its increment. Since by hypothesis (ii) $u$ is continuously invertible it follows that

$$
u_{2} \rightarrow u_{0} \Rightarrow x_{2} \rightarrow x_{0} \Rightarrow x_{1} \rightarrow x_{0},
$$

and since $\Phi$ is continuously differentiable we can choose, using (12), the value of $k$ to be sufficiently small that

$$
\begin{aligned}
\delta_{x} \Phi\left(\left(x_{0}, z_{2}^{*}\right) ; x_{1}-x_{0}\right)-\delta_{x} \Phi & \left(\left(x_{0}+k\left(x_{1}-x_{0}\right)\right) ; x_{1}-x_{0}\right) \\
& +z_{1}^{*}\left(g\left(x_{0}\right)\right)-z_{0}^{*}\left(g\left(x_{0}\right)\right)+u_{1}-u_{0} \leq 0 .
\end{aligned}
$$

Hence

$$
\Phi\left(x_{2}, z_{2}^{*}\right)-\delta_{x} \Phi\left(\left(x_{2}, z_{2}^{*}\right) ; x_{2}\right) \leq \Phi\left(x_{0}, z_{0}^{*}\right)-\delta_{x} \Phi\left(\left(x_{0}, z_{0}^{*}\right) ; x_{0}\right)
$$

which contradicts hypothesis (iii).

Therefore $\left(x_{0}, z_{0}^{*}\right)$ is the optimum solution of problem (6), (7), (8).

An equivalent form of this problem is:

$$
\begin{array}{ll}
\text { maximise } & -y_{0}^{*}\left(f\left(x_{0}\right)\right)-z^{*}\left(g\left(x_{0}\right)\right)-u^{0} \\
\text { subject to } & u^{0} \geqq 0 \\
\text { and } & z^{*} \geqq 0
\end{array}
$$


and since it has the solution $\left(x_{0}, z_{0}^{*}\right)$ there exists, by theorem $1, \xi_{0}^{*}$ such that $\left(\left(x_{0}, z_{0}^{*}\right) ; \xi_{0}^{*}\right)$ is optimal in the dual problem:

$$
\text { minimise }-y_{0}^{*}\left(f\left(x_{0}\right)\right)
$$

subject to

$$
-\delta_{\left(z^{*}, u^{0}\right)}\left[-z^{*}\left(g\left(x_{0}\right)\right)-u^{0}+\xi^{*}\left(u^{0}\right) ;\left(z^{*^{1}}, u^{0^{1}}\right)\right] \geqq 0
$$

and

$$
\xi^{*} \geqq 0
$$

for all

$z^{*^{1}} \geqq 0$

and all

$u^{0^{1}} \geqq 0$.

The inequality (29) can be written

$$
-\delta_{z^{*}}\left[-z^{*}\left(g\left(x_{0}\right)\right) ; z^{*^{1}}\right]-\delta_{u^{0}}\left[-u^{0}+\xi^{*}\left(u^{0}\right) ; u^{0^{1}}\right] \geqq 0 .
$$

Since the two terms in (33) are independent, they must individually satisfy the inequality; it follows that

$$
z^{*^{1}}\left(g\left(x_{0}\right)\right) \geqq 0
$$

which together with (31) implies that

$$
g\left(x_{0}\right) \geqq 0 .
$$

Hence

$$
x_{0} \in P_{x} \cap g^{-1}\left(P_{z}\right) .
$$

Equating the objective functions (25) and (28) at their extreme values, we have, by theorem 1 ,

$$
z_{0}^{*}\left(g\left(x_{0}\right)\right)+\delta_{x} \Phi\left(\left(x_{0}, z_{0}^{*}\right) ; x_{0}\right)=0 .
$$

Hence by (31) and (32) each term in (37) is zero:

$$
z_{0}^{*}\left(g\left(x_{0}\right)\right)=0
$$

and

$$
\delta_{x} \Phi\left(\left(x_{0}, z_{0}^{*}\right) ; x_{0}\right)=0 .
$$

From (31), (32), (38), and (39) it follows that $\Phi$ has a non-negative quasisaddle-point at $\left(x_{0}, z_{0}^{*}\right)$.

Hence by Theorem V. 0 of [2] (Kuhn-Tucker Theorem 3) $x_{0}$ maximises $y_{0}^{*}(f(x))$ over $y_{0}^{*}\left(f\left(P_{x} \cap g^{-1}\left(P_{z}\right)\right)\right)$.

\section{References}

[1] Duffin, R. J., "Infinite Programs", Linear Inequalities and Related Systems, Princeton University Press, 1956.

[2] Hurwicz, L., "Progranming in Linear Spaces", Studies in Linear and Non-Linear Programming, Stanford University Press, California, 1958. 
[3] Kuhn, H. W. and A. W. Tucker, "Non-linear programming", Proceedings of the Second Berkeley Symposium on Mathematical Statistics and Probability University of California Press, 1951.

[4] Karlin, S., "Mathematical Methods and Theory in Games, Programming and Economics", Addison-Wesley Publ. Co., Inc., Mass., 1959.

[5] Dennis, J. B., "Mathematical Programming and Electrical Networks", Technology Press and John Wiley and Sons, New York, 1959.

[6] Dorn, W. S., "Duality in Quadratic Programming", Q. Appl. Math., 18, 155-162, July 1960.

[7] Dorn, W. S., "A Duality Theorem for Convex Programs", I.B.M. Journal, 4, 407-413, October 1960.

[8] Wolfe, P., "A Duality Theorem for Non-linear Programming", Q. Appl. Math., 19. $239-244,1961$.

[9] Hanson, M. A., "A Duality Theorem in Non-linear Programming with Non-linear Constraints", Austral. J. Statistics, 3, 64_-72, 1961.

Department of Statistics

University of New South Wales

Sydney. 